\title{
Causas de la poca recuperación de valor de los residuos de aparatos eléctricos y electrónicos en Bogotá
}

\section{Causes of little value recovery of waste electrical and electronic equipment in Bogotá}

\author{
págs. 55-68 \\ Grupo de Investigación: CINDE \\ Línea de investigación: Logística y Gestión de Redes de Valor \\ Mónica Yinette Suárez Serrano• Jaime Leonardo Tarazona Silva•• Paula-Alejandra Triviño Quintero•.•
}

Recibido: agosto 01 de 2016

\section{RESUMEN}

El constante aumento en el consumo de dispositivos eléctricos y electrónicos (RAEE tipo 3) tales como computadores, periféricos y celulares; debido al exponencial desarrollo tecnológico que ha tenido esta industria en los últimos años y las diferentes estrategias que utilizan las empresas para que el consumidor considere obsoleto un dispositivo que cumple con todas las características funcionales; provoca un aumento en los niveles de los residuos de los RAEE tipo 3 que al no ser gestionados adecuadamente generan impactos negativos tanto al medio ambiente como a la sociedad. Por tanto, esta investigación tiene como objetivo identificar las causas de la baja recuperación del valor de este tipo de residuos en la ciudad de Bogotá. Dicha identificación de causas se realizó a través de fuentes de información secundaria y una posterior validación a través de fuentes de información primaria; esta última realizada mediante encuestas dirigidas a los diferentes actores de la cadena de suministros de celulares, computadores y periféricos de la ciudad de Bogotá, para posteriormente realizar un análisis de convergencia entre los resultados encontrados a través de la fuentes de información utilizadas, para así definir las causas de la problemática planteada.

Palabras clave: RAEE y Recuperación de Valor

\section{Aceptado: noviembre 24 de 2016}

Key words: WEEE and Recovery Value

The steady increase in the consumption of electrical and electronic equipment (WEEE Type 3 ) such as computers, peripherals and cellular; due to the exponential technological development that has taken this industry in recent years and the different strategies that companies use for the consumer to consider obsolete a device that meets all functional characteristics; It causes an increase in levels of WEEE waste type 3 not being managed properly generate negative both the environment and society impacts. Therefore, this research aims to identify the causes of the low recovery of the value of this type of waste in the city of Bogota. This identification of causes was made through secondary sources of information and further validation through primary information sources; the latter conducted through surveys directed at the different actors in the supply chain of cell phones, computers and peripherals Bogota, later to make an analysis of convergence between the results found through the sources of information used in order to define the causes of the issues raised.

- Ingeniera Industrial, Docente Investigadora. Universidad de América, monica.suarez@investigadores.edu.co - Ingeniero Industrial, Coinvestigador, Universidad de América, jaime.tarazona@estudiantes.uamerica.edu.co

..• Ingeniero Industrial, Coinvestigador, Universidad de América, paula.trivino@estudiantes.uamerica.edu.co 


\section{INTRODUCCIÓN}

En la actualidad el desarrollo de los países se encuentra enfocado principalmente en las tecnologías de la información y comunicación, por medio de las cuales se busca un desarrollo tanto social como económico (Blaser, 2009). Es por esto que el constante aumento en el consumo de este tipo de tecnologías es cada vez mayor, reflejándose en países como Colombia que se encuentran en vía de desarrollo (Silva, 2010). Este aumento trae como consecuencia directa el incremento en los volúmenes de residuos de aparatos eléctricos y electrónicos (RAEE), los cuales presentaron un crecimiento del 56\% en la generación per cápita del año 2008 al 2013 (Blaser, 2009).

Esta investigación se centra en la clasificación tipo 3 de los RAEE que corresponden a computadores, periféricos y celulares; residuos que requieren de un tratamiento específico y organizado para proteger el medio ambiente y la salud de la población (Blaser, 2009), por tanto surge el interrogante del por qué se dificulta la recuperación de valor presente en este tipo de residuos en la ciudad de Bogotá; tomando como antecedente que en países en desarrollo como Colombia éstos son gestionados principalmente por personas de bajos recursos que carecen de la capacitación especializada para esta operación (Colombia. Ministerio de Ambiente, Vivienda y Desarrollo Territorial, 2009) Es así, como a través de esta investigación se realiza un diagnóstico donde se identifican las diversas causas de la problemática planteada; las cuales sirven como referente para diseño de un modelo de logística inversa.

\section{MÉTODO}

El diagnostico se realizó en dos fases: en la primera de ellas se identificaron las causas asociadas a la poca recuperación de valor de los RAEE tipo 3 a través de la revisión y el análisis de fuentes secundarias. Dichas causas se esquematizaron en un diagrama de Ishikawa mixto en el que se combinaron las técnicas de las 6M y de estratificación (Gutiérrez Pulido \& Salazar, 2004). Una vez definidas las causas se incorporó la metodología de la Matriz Vester para la sistematización y priorización del problema focal (Martínez, 2009). En la segunda fase; se procedió a realizar la respectiva validación a través de la aplicación de encuestas dirigidas a diferentes actores de la cadena de suministro o entidades que se encuentran ligadas con las causas identificadas, para ello se definieron poblaciones y muestras aplicando métodos probabilísticos y no probabilísticos (Martínez C., 2000). Una vez tabulados y analizados los resultados de las encuestas se procedió a realizar un análisis de convergencia entre los resultados provenientes de las fuentes de información primaria y secundaria.

\section{Fundamentos teóricos}

A continuación se presenta una síntesis de los principales fundamentos teóricos que apoyan la investigación.

a. RAEE. Por sus siglas significa los residuos de aparatos eléctricos y electrónicos, son aquellos aparatos que ya han culminado o cumplido su vida útil, ya sea por la obsolescencia percibida de las personas o la programada por parte de las empresas; es decir aquellos que ya están dañados o son considerados obsoletos por el común y son desechados, y que requieren electricidad para su funcionamiento. Hay un sin número de aparatos que hacen parte de los RAEE, debido al constante crecimiento de la tecnología; es por esto que los residuos de aparatos eléctricos y 
LÍNEA DE INVESTIGACIÓN: LOGÍSTICA Y GESTIÓN DE REDES DE VALOR

electrónicos se categorizan según su tamaño o funcionalidad (Ott, 2008). Esta investigación se centra en los RAEE clasificación tres, que son los que comprenden a los equipos de informática y comunicaciones como celulares, computadores y periféricos.

b. Problemática de los RAEE. Este tipo de residuos presentan un problema ambiental por los componentes que vienen asociados a estos residuos, pero también representan una oportunidad de negocio por la misma razón. Aunque los aparatos eléctricos y electrónicos no representan una amenaza mientras están siendo utilizados, una vez han cumplido su ciclo de vida y se convierten en RAEE es importante darle el manejo adecuado a estos materiales, sobre todo en los países en vía de desarrollo ya que en estos, los encargados de dicha gestión son personas de bajos recursos, por ende generan mayores impactos sociales y ambientales debido al desconocimiento sobre el desensamble especializado que deben tener estos residuos. Por otra parte la cantidad de material que se puede recuperar y los costos asociados a estos son representativas ya que en "230 millones de computadores y 1000 millones de teléfonos celulares es posible la recuperación de 70 toneladas de oro y 535 de plata", cifras que representan para cada uno de estos metales el 3\% de la producción minera mundial (Colombia. Ministerio de Ambiente, Vivienda y Desarrollo Territorial, 2009).

\section{RESULTADOS}

Tras la revisión de diversas fuentes de información secundaria, como lo fueron artículos científicos, tesis, estudios y reportes relacionados con las dificultades que existen en el país para la recuperación de valor presente en los RAEE tipo tres; se realiza la construcción del diagrama de Ishikawa y una posterior priorización de causas para que estas puedan ser validadas a través de encuestas dirigidas a los diferentes actores que se encuentran relacionadas con ellas y finalmente adaptar las causas relacionadas con la problemática de la investigación.

\section{Construcción del diagrama de Ishikawa}

Con la construcción del diagrama de Ishikawa se pretenden identificar las principales causas incidentes en el problema central de la investigación, definido como ¿por qué se dificulta la recuperación de valor presente en los RAEE tipo 3 en la ciudad de Bogotá?. Este diagrama se elaboró con base en la revisión de fuentes de información secundaria en donde después de su análisis, los investigadores identificaron diversas causas principales del problema y sub causas; construyendo así un diagrama de Ishikawa mixto entre el método de las "6M's" y el estratificado, las causas identificadas se presenta en la Figura 1.

Los siguientes son los aspectos más relevantes de cada una de causas principales identificadas:

a. Materiales. Los materiales que conforman los RAEE tipo 3, pueden ser tanto valiosos como peligrosos. Los materiales peligrosos presentes en este tipo de aparatos se encuentran por lo general de forma sólida no dispersable, por lo tanto no representan un riesgo para la salud humana en el uso habitual de estos dispositivos; pero al momento de terminar su ciclo de vida y ser sometidos a algún proceso de recuperación emperico pueden generar emisiones y causar daños tanto al personal que lo manipula como al ambiente si no se tratan de manera adecuada. 


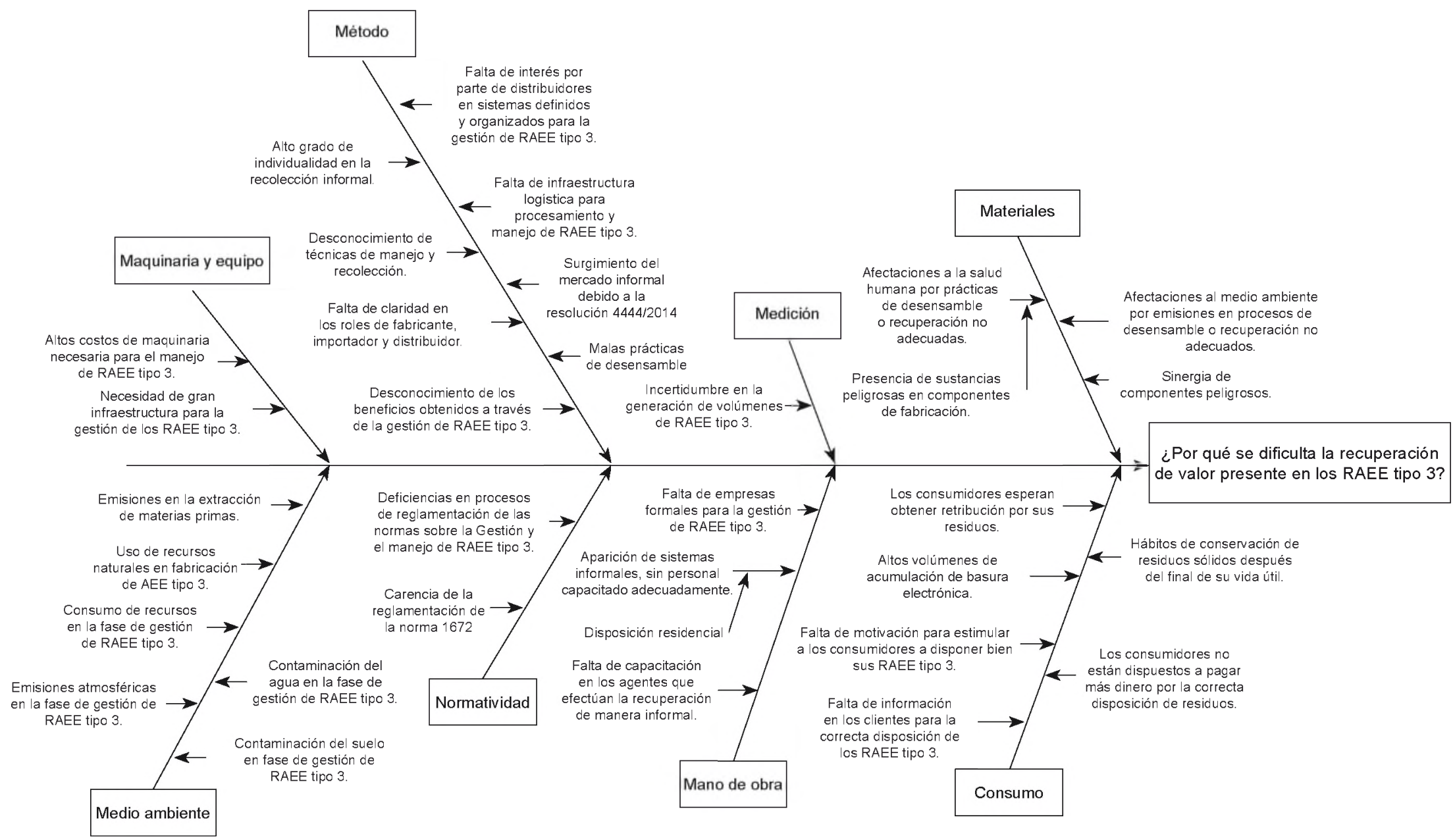


LÍNEA DE INVESTIGACIÓN: LOGÍSTICA Y GESTIÓN DE REDES DE VALOR

De igual manera cabe resaltar que el porcentaje de sustancias peligrosas que componen a los RAEE es del $0,5 \%$ del total del material recogido. Por lo anterior, es evidente que un solo equipo de este tipo de residuo no se considera como un residuo peligroso; sin embargo estos son catalogados como tal gracias a la sinergia que forma esta pequeña proporción en grandes cantidades o volúmenes de estos desechos. Dentro de elementos peligrosos que se encuentran presentes en los RAEE tipo tres se destacan los compuestos halógenos como los retardantes de llama, los metales pesados el arsénico, bario, cadmio, etc y otros elementos como el sulfuro de zinc y sustancias radioactivas como americio. (Colombia. Ministerio de Ambiente, Vivienda y Desarrollo Territorial, 2009).

b. Método. En cuanto a las subcausas relacionadas con la "rama" de método podemos encontrar que: en Colombia no se cuenta con la infraestructura logística necesaria para el procesamiento y manejo de este tipo de residuos. Los pocos fabricantes nacionales y los grandes distribuidores de este tipo de aparatos electrónicos no tienen un sistema de recolección definido y organizado; por lo que de igual manera incrementa el número de recolectores informales, los cuales no tienen los conocimientos necesarios para realizar esta labor (Ott, 2008).

De igual manera el poco conocimiento que se tiene acerca del manejo de estos residuos, de las técnicas de gestión aplicables a éstos y la falta de interés y compromiso por parte de los actores involucrados a lo largo de la cadena de suministro, contribuyen a la no recuperación de valor de este tipo de residuos (Colombia. Ministerio de Ambiente, Vivienda y Desarrollo Territorial, 2009).

En cuanto a los beneficios económicos de la recuperación de los residuos en estudio, se reflejan en el ahorro de la compra de materias primas vírgenes. En el caso de los RAEE, se estima que podrían extraerse USD 21 mil millones al año en la recuperación de mátales preciosos que se encuentran presentes en estos residuos. En el caso Colombiano, y según estimaciones realizadas por el EMPA, para el año 2007 se encontraron 45.000 toneladas de residuos de computadores en el país, las cuales contienen alrededor de 3.000 toneladas de cobre; con lo que a precios del cobre a principios del 2008 equivaldría a 25 millones de USD (Romero, 2014).

El cumplimiento de la normatividad, se refiere a los requerimientos legales establecidos en cada país, en cuanto a la responsabilidad de los productos, los procesos de producción o distribución; con el fin de disminuir los impactos ambientales y sociales que estos traigan a lo largo de su realización y disposición final. En relación con la responsabilidad social se encuentra el valor agregado que el cliente le atribuye al producto gracias a la conciencia ambiental que toma la empresa a lo largo del ciclo de vida de este; y que va acorde con la conciencia ambiental del cliente (Roman, 2015).

Otra razón por la cual se dificulta la recuperación de valor presente en los RAEE tipo tres radica en que no se tiene total claridad en los roles de fabricante, importador y distribuidor de este tipo de aparatos, en cuanto al concepto de responsabilidad extendida del productor. Esto se da ya que en Colombia no existe ningún fabricante de este tipo de aparatos, por lo que los demás eslabones no saben quién debe asumir la responsabilidad de los RAEE tipo tres. Actualmente en la industria de la telefonía móvil los operadores son los que han tomado el rol de "fabricante" a nivel nacional, encargándose de la operatividad y responsabilidad económica en la gestión de los RAEE generados por sus clientes (Roman, 2015). 
Una vez identificadas cada una de las causas y sub causas del problema central, las cuales se encuentran reflejadas en el diagrama de Ishikawa, se prosigue a la priorización de cada una de ellas.

c. Maquinaria y equipo. Las causas encontradas en esta "rama" se ven asociadas con los costos de la maquinaria y equipos auxiliares que son necesarios y esenciales para la realización de las labores de recolección y posteriores procesos de desensamble para la descomposición final del residuo y obtención de materiales. La infraestructura requerida para una planta de manejo de RAEE consta del terreno, maquinas, equipos de apoyo, medios de transporte, equipos de seguridad industrial y protección ambiental; los cuales en conjunto son un obstáculo para el tratamiento de los RAEE debido a los altos costos de puesta en marcha de dicha planta (Kenny, 2013).

d. Mano de Obra. Las causas encontradas en la "rama" de mano de obra se relacionan con la falta de empresas $u$ organizaciones que se dediquen formalmente al tratamiento de los RAEE y al contrario de esta situación se presenta un crecimiento de actividades artesanales de recuperación de RAEE, bajo sistemas informales los cuales no garantizan la seguridad de quienes trabajan allí frente a la exposición y manera de grandes volúmenes de material toxico (Ott, 2008). De igual manera es importante resaltar que las personas que conforman este gremio de recolección informal son personas las cuales basan su principal fuente de ingresos en la realización de estas actividades; por lo cual es difícil que la informalidad de estas actividades se disminuyan y por el contrario, como se ha visto en los últimos años, esta seguirá en aumento (Daniel, 2009) .

e. Consumo. En cuanto a las causas relacionadas con la "rama" de consumo, se encuentra que la responsabilidad del consumidor en las labores necesarias para la recuperación de valor de los RAEE tipo tres es esencial; ya que este es el último eslabón o actor con el cual el producto hace contacto en la cadena de suministro y por tanto, es el encargado de realizar la correcta disposición de los residuos para implementar sistemas de recolección eficientes. En esta causa identificada en la problemática planteada se deben tener en cuenta aspectos como el periodo de vida útil que tanto los usuarios privados como los corporativos le dan a los Aparatos eléctricos y electrónicos AEE según sus hábitos de consumo. En este caso los consumidores no suelen disponer adecuadamente este tipo de residuos después de que ya no les son útiles, contrario a esto suelen guardarlos por periodos de tiempo sin darles ningún uso o regalarlo a personas cercanas que tal vez los necesiten; dificultando de esta manera la recuperación de estos residuos para someterlos a procesos de recuperación de valor (Ott, 2008).

f. Normatividad. Las causas que se pueden encontrar en la "rama" de normatividad se relacionan con las leyes Colombianas vigentes a la fecha, en cuanto a la gestión de residuos sólidos y la responsabilidad extendida del productor. Actualmente la normatividad existente en este ámbito no es lo suficientemente estricta para obligar a los productores o distribuidores a responsabilizarse de los desechos que generan los productos que ellos ponen en el mercado. Aunque actualmente se encuentra aprobada la ley 1672, en la cual se especifican las responsabilidades extendidas de los diferentes actores de la cadena de suministro, con el fin de obligarlos a gestionar sus residuos, aún está en proceso de reglamentación, ya que no se encuentran definidos los detalles de cumplimiento, obligaciones y penalidades, esto implica que los actores de la cadena de los AEE tipo 3 aún no se hagan cargo de sus residuos (Alcaldía Mayor de Bogotá, 2008)

g. Medio Ambiente. Disponer de este tipo de RAEE en tan poco tiempo, pues su ciclo de vida oscila entre los 4 y 5 años, no es nada amigable con el medio ambiente, por eso se debe consi- 
LÍNEA DE INVESTIGACIÓN: LOGÍSTICA Y GESTIÓN DE REDES DE VALOR

derar la reutilización de estos RAEE, volviendo un poco más rentable el consumo de energía en términos ambientales. En la fase de post-consumo, la gestión de estos RAEE representan efectos negativos en muchos aspectos como: impactos ambientales, efectos nocivos en la salud de las personas, baja rentabilidad, y esto se debe a que son dispositivos compactos con alta presencia de materiales peligrosos (Grupo de investigación CIT, 2015).

Debido a las sustancias nocivas que están presentes en los diferentes componentes que conforman a lo RAEE tipo 3 (bifenilos policlorados, Éteres bifenilicos, materiales tóxicos o materiales pesados), se generan grandes cantidades de emisiones al medio ambiente cuando son incinerados de manera incorrecta, promoviendo la formación de sustancias toxicas como los son las dioxinas y los furanos. A su vez "este tipo de equipos contiene materiales tóxicos como plomo $(\mathrm{Pb})$, azufre $(\mathrm{S})$ y níquel $(\mathrm{Ni})$, que puedan representar un daño ambiental significativo si son desechados en rellenos sanitarios ordinarios", estas sustancias peligrosas al ser liberadas al ambiente, representan un peligro de contaminación de suelos, aire y agua. Cabe resaltar que si estos residuos son dispuestos en rellenos sanitarios sin ningún tipo de tratamiento generan impactos ambientales, debido al proceso de lixiviación, "La lixiviación es el proceso químico por medio del cual se extraen los componentes de una muestra a partir de un líquido como el ácido acético o el ácido sulfúrico" (Luis, 2010).

h. Medición. Para el caso de esta espina, se identifica un alto grado de incertidumbre en cuanto a la predicción de la demanda de residuos que puedan entrar al sistema logístico inverso de gestión de RAEE tipo 3, esta situación se da básicamente por dos razones principales, en este sentido la primera es que los consumidores no disponen de manera adecuada estos residuos una vez finalizado su ciclo de vida, en contraste los conservan por largos periodos de tiempo dificultando el proceso de recuperación de los mismos. La segunda razón que también es relevante es que los consumidores ven estos equipos que ya finalizaron su ciclo de vida como una inversión, esto debido a su alto valor de adquisición, por la cual esperan recibir una retribución lo que genera más hábitos de conservación o disposición inadecuada, esto también se da porque los clientes no están dispuestos a pagar algo de más por la correcta disposición de este tipo de residuos.

\section{Matriz Vester}

Es importante realizar el estudio de priorización, ya que éste permite: la identificación de las causas más incidentes en el problema de la investigación, evitar posibles redundancias y descartar causas que no sean importantes para el diagnóstico. El estudio de priorización para las causas ya definidas en el diagrama de Ishikawa se realiza a través de la matriz de Vester, pues, ésta permite el análisis de la información obtenida, para elaborar a partir de allí, herramientas de recolección primaria basadas en las causas críticas y activas según los resultados de la matriz. De la priorización se obtiene que del total de 33 causas identificadas, el $40 \%$ son activas, el $27 \%$ pasivas, el $18 \%$ críticas y el $15 \%$ indiferentes, así como se muestra en la Figura 1 . Adicionalmente en las Tablas 1 y 2 , se clasifican cada una de las causas en los cuadrantes según su importancia e incidencia en el problema central.

Con base a la priorización de causas realizada por medio de la matriz de Vester, se centra la investigación en las causas críticas y activas; ya que según esta metodología las causas activas son las que influencian las demás causas y las causas críticas son las que se ven más influenciadas por las demás. En consecuencia, como resultado de esta Fase el estudio se segmenta a un total de 19 causas; 13 activas y seis críticas. 


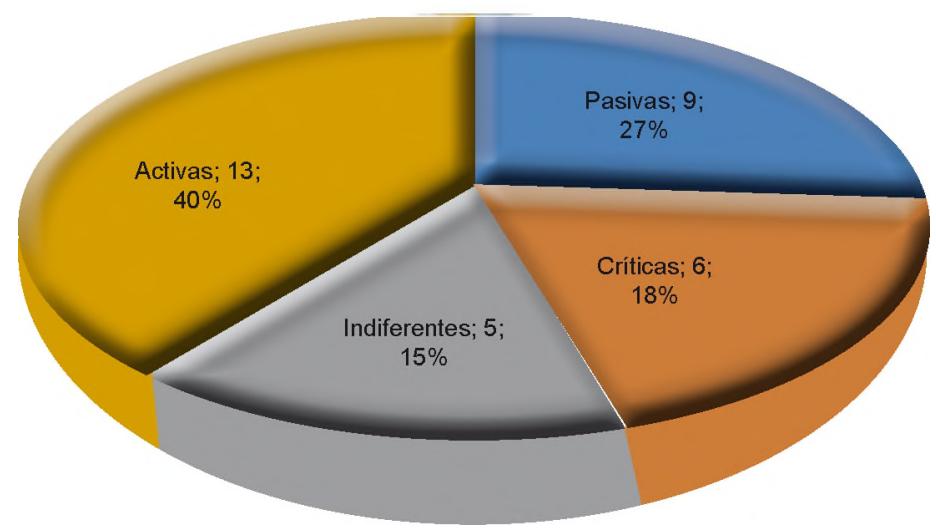

Figura 2. Priorización de causas

Fuente: Autores

\section{Recolección de información de fuentes primarias}

Partiendo del diagnóstico anterior, se procede a validar las causas activas y críticas a través de encuestas dirigidas a los actores que se reconocieron están ligados a las causas identificadas, definiendo para cada uno de ellos la población y la muestra para el estudio. Cabe resaltar que para la selección de esta última se realizó un muestreo mixto, empleando técnicas probabilísticas y no probabilísticas. En la Figura 2 se sintetiza la definición de las poblaciones y muestras correspondientes a cada actor de la cadena de suministros de los AEE. La elaboración de los cuestionarios se basó en la estructuración de preguntas que permitieran validar las causas críticas y activas identificadas anteriormente a través de la Matriz Vester.

Tabla 1.

Matriz de Vester. Causas pasivas y críticas

\begin{tabular}{clcl}
\hline $\begin{array}{c}\text { No. } \\
\text { causa }\end{array}$ & \multicolumn{1}{c}{ Causas pasivas } & $\begin{array}{c}\text { No. } \\
\text { causa }\end{array}$ & \multicolumn{1}{c}{ Causas críticas } \\
\hline $8 \quad \begin{array}{l}\text { Afectaciones a la salud humana } \\
\text { por prácticas de desensamble } \\
\text { recuperación inadecuadas. }\end{array}$ & 3 & $\begin{array}{l}\text { Falta de infraestructura logística para } \\
\text { procesamiento y manejo de RAEE tipo 3. }\end{array}$ \\
& $\begin{array}{l}\text { Afectaciones al medio ambiente } \\
\text { por emisiones en procesos de } \\
\text { desensamble o recuperación no } \\
\text { adecuados. }\end{array}$ & 5 & $\begin{array}{l}\text { Falta de interés por parte de distribuidores en } \\
\text { sistemas definidos y organizados para la gestión } \\
\text { de RAEE tipo 3. }\end{array}$ \\
$11 \quad \begin{array}{l}\text { Sinergia de componentes peligrosos. } \\
13 \quad\end{array}$ & 21 & $\begin{array}{l}\text { Falta de empresas formales para el tratamiento } \\
\text { de los RAEE tipo 3. }\end{array}$ \\
fabricación de AEE tipo 3. & 22 & $\begin{array}{l}\text { Aparición de sistemas informales, sin personal } \\
\text { capacitado adecuadamente. }\end{array}$ \\
\hline
\end{tabular}




\begin{tabular}{clll}
\hline $\begin{array}{c}\text { No. } \\
\text { causa }\end{array}$ & \multicolumn{1}{c}{ Causas pasivas } & $\begin{array}{c}\text { No. } \\
\text { causa }\end{array}$ & \multicolumn{1}{c}{ Causas críticas } \\
\hline 14 & $\begin{array}{l}\text { Consumo de recursos en la fase de } \\
\text { gestión de los RAEE tipo 3. }\end{array}$ & & \\
15 & $\begin{array}{l}\text { Emisiones atmosféricas en fase de } \\
\text { gestión de RAEE tipo 3. }\end{array}$ & 29 & $\begin{array}{l}\text { Altos volúmenes de acumulación de basura } \\
\text { electrónica. }\end{array}$ \\
$16 \quad \begin{array}{l}\text { Contaminación del agua en fase de } \\
\text { gestión de RAEE tipo 3. }\end{array}$ & 33 & $\begin{array}{l}\text { Falta de información en los clientes para la } \\
\text { correcta disposición de los RAEE tipo 3 }\end{array}$ \\
$17 \quad \begin{array}{l}\text { Contaminación del suelo en fase de } \\
\text { gestión de RAEE tipo 3 }\end{array}$ & & \\
\hline 11 & Malas prácticas de desensamble & & \\
\hline
\end{tabular}

Fuente: Autores

Tabla 2.

Matriz de Vester. Causas indiferentes y Activas

\begin{tabular}{|c|c|c|c|}
\hline $\begin{array}{l}\text { No. } \\
\text { causa }\end{array}$ & Causas indiferentes & $\begin{array}{l}\text { No. } \\
\text { causa }\end{array}$ & Causas activas \\
\hline 1 & $\begin{array}{l}\text { Altos costos de maquinaria necesaria } \\
\text { para el manejo de RAEE. }\end{array}$ & 4 & $\begin{array}{l}\text { Desconocimiento de técnicas de manejo y } \\
\text { recolección. }\end{array}$ \\
\hline 2 & $\begin{array}{l}\text { Necesidad de gran infraestructura para } \\
\text { la gestión de los RAEE tipo } 3 \text {. }\end{array}$ & 6 & $\begin{array}{l}\text { Desconocimiento de los beneficios obtenidos a } \\
\text { través de la gestión de RAEE tipo } 3 \text {. }\end{array}$ \\
\hline 12 & $\begin{array}{l}\text { Emisiones en la extracción de materias } \\
\text { primas. }\end{array}$ & 7 & $\begin{array}{l}\text { Falta de claridad en los roles de fabricante, } \\
\text { importador y distribuidor. }\end{array}$ \\
\hline 9 & $\begin{array}{l}\text { Presencia de sustancias peligrosas en } \\
\text { componentes de fabricación. }\end{array}$ & 18 & $\begin{array}{l}\text { Carencia en la normatividad sobre la gestión y el } \\
\text { manejo de RAEE tipo } 3 \text {. }\end{array}$ \\
\hline \multirow[t]{7}{*}{32} & $\begin{array}{l}\text { Incertidumbre en la generación de } \\
\text { volúmenes de RAEE tipo } 3\end{array}$ & 19 & $\begin{array}{l}\text { Surgimiento del mercado informal debido a la } \\
\text { resolución } 4444 / 2014 \text {. }\end{array}$ \\
\hline & & 20 & $\begin{array}{l}\text { Carencia de la reglamentación de la norma } \\
1672 .\end{array}$ \\
\hline & & 23 & Disposición residencial. \\
\hline & & 24 & $\begin{array}{l}\text { Alto grado de individualidad en la recolección } \\
\text { informal. }\end{array}$ \\
\hline & & 25 & $\begin{array}{l}\text { Falta de capacitación en los agentes que } \\
\text { efectúan la recuperación de manera informal. }\end{array}$ \\
\hline & & 26 & $\begin{array}{l}\text { Hábitos de conservación de residuos después } \\
\text { del final de su vida útil. }\end{array}$ \\
\hline & & 27 & $\begin{array}{l}\text { Los consumidores no están dispuestos a pagar } \\
\text { más dinero por la correcta disposición de } \\
\text { residuos. }\end{array}$ \\
\hline
\end{tabular}




\begin{tabular}{|c|c|c|c|}
\hline $\begin{array}{l}\text { No. } \\
\text { causa }\end{array}$ & Causas indiferentes & $\begin{array}{c}\text { No. } \\
\text { causa }\end{array}$ & Causas activas \\
\hline & & 28 & $\begin{array}{l}\text { Los consumidores esperan obtener retribución } \\
\text { por sus residuos. }\end{array}$ \\
\hline & & 30 & $\begin{array}{l}\text { Falta de motivación para estimular a los } \\
\text { consumidores a disponer bien sus RAEE tipo } 3 .\end{array}$ \\
\hline
\end{tabular}

Fuente: Autores

\section{Validación de la información}

Partiendo del análisis y tabulación de datos arrojados por las encuestas, se prosigue a realizar la validación de la información mediante un análisis de convergencia; en donde se realiza una comparación entre la información obtenida con fuentes de información secundaria y la obtenida con fuentes primarias. De esta forma, si los resultados arrojados por las fuentes de información utilizadas, coinciden, se considera que existe convergencia en las causas analizadas; por lo tanto la(s) causa(s) serán validadas. Si la información obtenida por las dos fuentes, tanto primaria como secundaria, llegase a tener resultados diferentes se llega a la conclusión que no existe convergencia; en este caso se asumirá como causa la información obtenida por fuentes de información primaria.

Por otro lado, cabe mencionar que en el diseño de las encuestas se contemplaron causas inferidas por los investigadores, que aunque no se contemplaron en el diagrama Ishikawa se pretendía validar su pertinencia a través de la consulta de la fuentes primarias. Por tanto, para este tipo de causas no aplica el análisis de convergencia, ya que no tienen raíces en la primera fase del diagnóstico, no obstante aquellas que se validaron hacen parte del grupo de causas identificadas en el diagnóstico realizado.

Finalmente con base a los resultados obtenidos en el análisis de convergencia se validaron el $54 \%$ de las causas activas, el $100 \%$ de causas críticas y el $66 \%$ de las inferidas por los investigadores. En la Tabla 3 se presentan las 29 causas que fueron identificadas y validadas ecomo resultado del diagnóstico realizado.

Tabla 3.

Resultados de la validación

\begin{tabular}{lll}
\hline ID & $\begin{array}{c}\text { Tipo de } \\
\text { causa }\end{array}$ & \multicolumn{1}{c}{ Causas validadas } \\
\hline 1 & Activas & Falta de claridad en los roles de fabricante, importador y distribuidor. \\
2 & Activas & Surgimiento del mercado informal debido a la resolución 4444/2014. \\
3 & Activas & Carencia de la reglamentación de la norma 1672. \\
4 & Activas & Disposición residencial. \\
5 & Activas & Falta de capacitación en los agentes que efectúan la recuperación de manera informal. \\
6 & Activas & Hábitos de conservación de residuos después del final de su vida útil. \\
\hline
\end{tabular}


LÍNEA DE INVESTIGACIÓN: LOGÍSTICA Y GESTIÓN DE REDES DE VALOR

\begin{tabular}{|c|c|c|}
\hline ID & $\begin{array}{c}\text { Tipo de } \\
\text { causa }\end{array}$ & Causas validadas \\
\hline 7 & Activas & Falta de motivación para estimular a los consumidores disponer bien sus RAEE tipo 3. \\
\hline 8 & Críticas & Falta de infraestructura logística para procesamiento y manejo de RAEE tipo 3. \\
\hline 9 & Críticas & $\begin{array}{l}\text { Falta de interés por parte de distribuidores en sistemas definidos y organizados para la } \\
\text { gestión de RAEE tipo } 3 \text {. }\end{array}$ \\
\hline 10 & Críticas & Falta de empresas formales para el tratamiento de los RAEE tipo 3. \\
\hline 11 & Críticas & Aparición de sistemas informales, sin personal capacitado adecuadamente. \\
\hline 12 & Críticas & Altos volúmenes de acumulación de basura electrónica. \\
\hline 13 & Críticas & Falta de información en los clientes para la correcta disposición de los RAEE tipo 3 \\
\hline 14 & Inferidas & Periodos de cambio de celulares y computadores. \\
\hline 15 & Inferidas & Localización de puntos estratégicos encaminados a la recolección de estos residuos. \\
\hline 16 & Inferidas & Existen convenios para la recolección de RAEE tipo 3. \\
\hline 17 & Inferidas & La recolección se da por agentes externos. \\
\hline 18 & Inferidas & Bonos de descuento como estrategias de recolección. \\
\hline 19 & Inferidas & Se identifica la posibilidad de redes de cooperación. \\
\hline 20 & Inferidas & Reciclaje informal procesa cantidades mínimas de RAEE tipo 3. \\
\hline 21 & Inferidas & $\begin{array}{l}\text { Reciclaje informal se caracteriza en actividades de recolección almacenamiento y } \\
\text { ventas. }\end{array}$ \\
\hline 22 & Inferidas & Reciclaje informal se enfoca en recuperar platicos, cobre, acero y vidrio. \\
\hline 23 & Inferidas & $\begin{array}{l}\text { Etapas presentes en la gestión de los RAEE tipo } 3 \text {, Recolección, recepción, } \\
\text { clasificación, identificación, almacenamiento, de manufactura, salidas o disposición } \\
\text { final. }\end{array}$ \\
\hline 24 & Inferidas & $\begin{array}{l}\text { Se caracterizan las siguientes actividades de gestión para el sector formal: Reusó, } \\
\text { reacondicionamiento, reparación, reciclaje. }\end{array}$ \\
\hline 25 & Inferidas & Para el reciclaje, la mayoría de casos se da manualmente. \\
\hline 26 & Inferidas & Convenios con empresas privadas. \\
\hline 27 & Inferidas & El producto final se vende se dona o se dispone. \\
\hline 28 & Inferidas & Salidas del producto se da por exportación o subastas. \\
\hline 29 & Inferidas & Se recuperan vidrio oro aluminio acero cobre y plásticos entre otros. \\
\hline
\end{tabular}

Fuente: Autores 


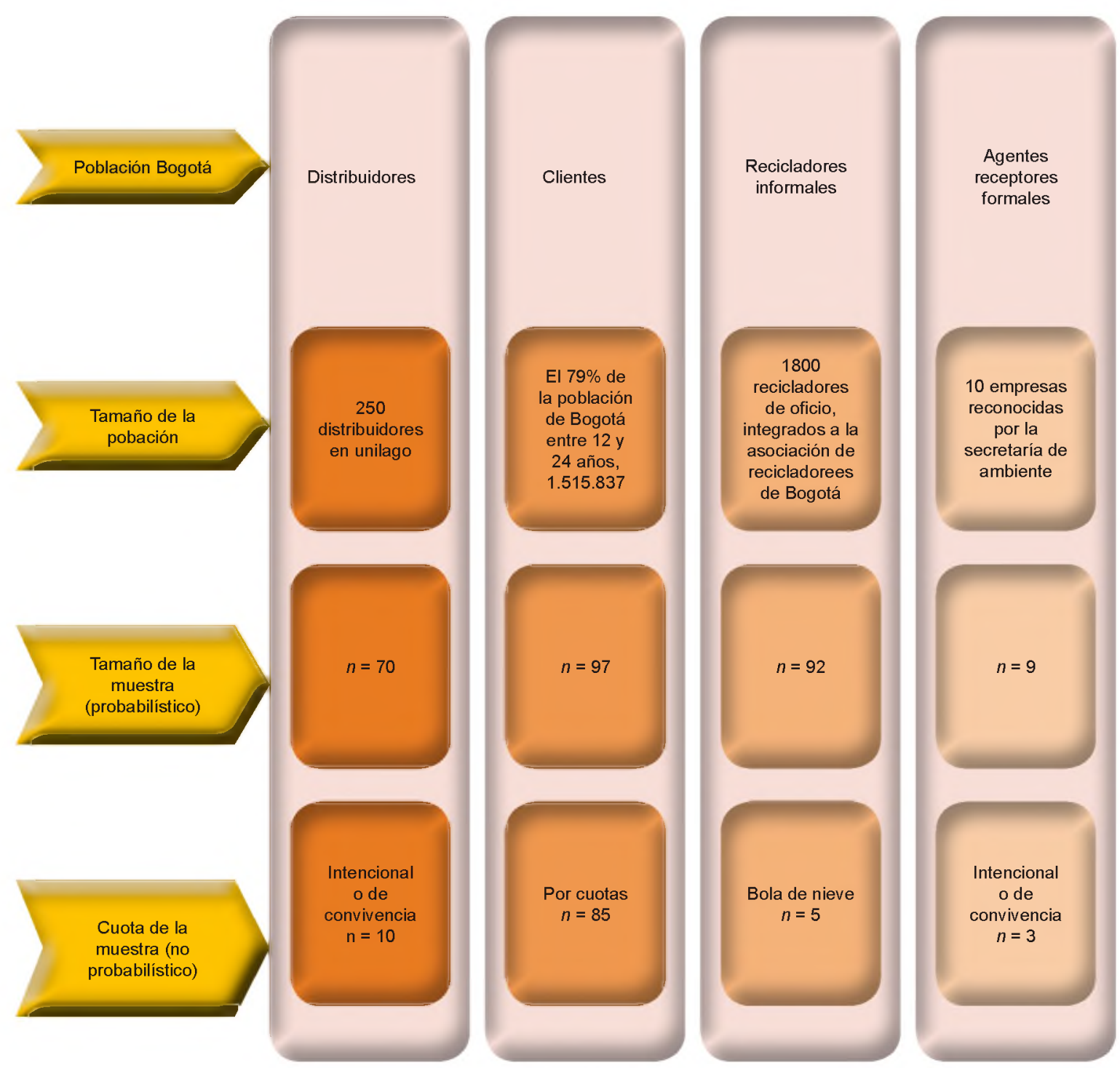

Figura 3. Definición de Poblaciones y muestras para los actores de la Cadena de Suministro de IOS AEE

Fuente: Autores

\section{CONCLUSIONES}

Al realizar la exploración y validación de información a través de fuentes de información primaria, se observa que no todas las causas encontradas en fuentes de información secundaria se reconocen por los actores que están directamente relacionados con ellas; por ello que se prioriza la información suministrada por los encuestados ya que ellos son quienes tienen el conocimiento y relación directa con la gestión de los RAEE.

Para el caso de las causas activas que no tuvieron convergencia en el análisis y por lo tanto no fueron validadas o reconocidas en esta investigación, es importante resaltar que éstas fueron iden- 
LÍNEA DE INVESTIGACIÓN: LOGÍSTICA Y GESTIÓN DE REDES DE VALOR

tificadas como comportamientos presentados años atrás y que fueron cambiando o desapareciendo a través del tiempo, razón por la cual, los actuantes de la cadena de los AEE no las reconocen en la actualidad.

A partir de este trabajo se proponen como trabajos futuros el diseño de herramientas de gestión logística que permitan mitigar las causas identificadas y aumentar la eficiencia y eficacia en la recuperación de valor presente en los RAEE tipo 3.

\section{REFERENCIAS}

Alcaldía Mayor de Bogotá. (2008). Proyecto de acuerdo No, 139 de 2008. Proyecto de acuerdo. Bogotá, Colombia. Obtenido de http://www.alcaldiabogota.gov.co/sisjur/normas/Norma1. jsp?i=29445. [Citado el 20 de Octubre de 2015].

Blaser, F. (2009). Diagnóstico de Electrodomésticos y de Aparatos Electrónicos de Consumo. Bogotá, Colombia: Swiss Federal Laboratories for Materials Testing and Research (Empa), Asociación Nacional de Empresarios de Colombia (ANDI).

Colombia. Ministerio de Ambiente, Vivienda y Desarrollo Territorial. (2009). Lineamientos técnicos para el manejo de residuos de aparatos eléctricos y electrónicos. Bogotá, Colombia: Ministerio de Ambiente, Vivienda y Desarrollo Territorial;.

Daniel, O. (Mayo de 2009). Manejo de los RAEE a través del sector informal en Medellín. Obtenido de Ewasteguide: http://ewasteguide.info/files/EMPA-CNPMLTA_Manejo_RAEE_SectorInformal_MDE.pdf

Grupo de investigación CIT. (Octubre de 2015). Residuos de Aparatos Eléctricos y Electrónicos. Obtenido de Círculo de Innovación y Tecnología: http://www2.uca.es/grup-invest/cit/RAEE.htm

Gutiérrez Pulido, H., \& Salazar, R. (2004). Control estadístico de la calidad y seis sigma. Guanajuato, México: Mc Graw Hill.

Kenny, S. (2013). Estudio de Factibilidad para el montaje de una planta de reciclaje de residuos eléctricos y electrónicos en Cartagena. Medellín, Colombia. Obtenido de https://repository. eafit.edu.co: https://repository.eafit.edu.co/bitstream/handle/10784/1467/FajardoSuarez_Kenny_2013.pdf?sequence=1\&isAllowed=y

Luis, H. (Diciembre de 2010). La basura electrónica y la contaminación ambiental. Enfoque UTE, 1(1), 46-61.

Martínez, C. (2000). Estadística y muestreo. Bogotá, Colombia: Eco Ediciones.

Martínez, M. (Enero de 2009). Diagnóstico de comunicación organizacional del Pedro Goméz y ClA. Bogotá, Colombia. 
Ott, D. (2008). Gestión de residuos electrónicos en Colombia. Diagnóstico de computadores y teléfonos celularea. Medellín: Centro Nacional de producción más limpia.

Roman , I. (2015). Ewaste en Colombia. GSMA. Obtenido de http://www.gsma.com/latinamerica/ wp-content/uploads/2015/02/ewaste-colombia.pdf

Romero, J. (Agosto de 2014). Colombia vs. la basura electrónica. Bogotá, Colombia.

Silva, U. (2010). Los residuos electrónicos: Un desafío para la sociedad del Conocimiento en América Latina y el Caribe. Montevideo, Uruguay: United Nations Educational, Scientific and Cultural Organization. 